\title{
Fluorine F 18 Fluorthanatrace
}

National Cancer Institute

\section{Source}

National Cancer Institute. Fluorine F18 Fluorthanatrace. NCI Thesaurus. Code C121942.

A radiotracer consisting of an analogue of the poly(ADP-ribose) polymerase 1 (PARP1)

inhibitor rucaparib radiolabeled with the positron emitting isotope fluorine $F 18$, which can potentially be used for the imaging of PARP1 expression using positron emission tomography (PET). Upon administration, fluorine $\mathrm{F} 18$ fluorthanatrace targ ets and binds to PARP1. Upon PET, PARP1-expressing tumor cells can be visualized. PARP1, which is overexpressed in many cancer cell types, catalyzes post-translational ADP-ribosylation of nuclear proteins and plays a key role in the repair of DNA strand breaks. 